\title{
Multiplicity formulas for fundamental strings of representations of classical Lie algebras
}

Emilio A. Lauret, and Fiorela Rossi Bertone

Citation: Journal of Mathematical Physics 58, 111703 (2017);

View online: https://doi.org/10.1063/1.4993851

View Table of Contents: http://aip.scitation.org/toc/jmp/58/11

Published by the American Institute of Physics

\section{Articles you may be interested in}

Rigged configuration descriptions of the crystals $B(\infty)$ and $B(\lambda)$ for special linear Lie algebras Journal of Mathematical Physics 58, 101701 (2017); 10.1063/1.4986276

A new algorithm for computing branching rules and Clebsch-Gordan coefficients of unitary representations of compact groups

Journal of Mathematical Physics 58, 101702 (2017); 10.1063/1.5004259

On the finite $\mathrm{W}$-algebra for the Lie superalgebra $\mathrm{Q}(\mathrm{N})$ in the non-regular case Journal of Mathematical Physics 58, 111701 (2017); 10.1063/1.4993709

$Z_{2} \times Z_{2}$ generalizations of $\mathcal{N}=2$ super Schrödinger algebras and their representations Journal of Mathematical Physics 58, 113501 (2017); 10.1063/1.4986570

Existence of topological multi-string solutions in Abelian gauge field theories Journal of Mathematical Physics 58, 111511 (2017); 10.1063/1.4997983

Three dimensional reductions of four-dimensional quasilinear systems Journal of Mathematical Physics 58, 111510 (2017); 10.1063/1.5006601

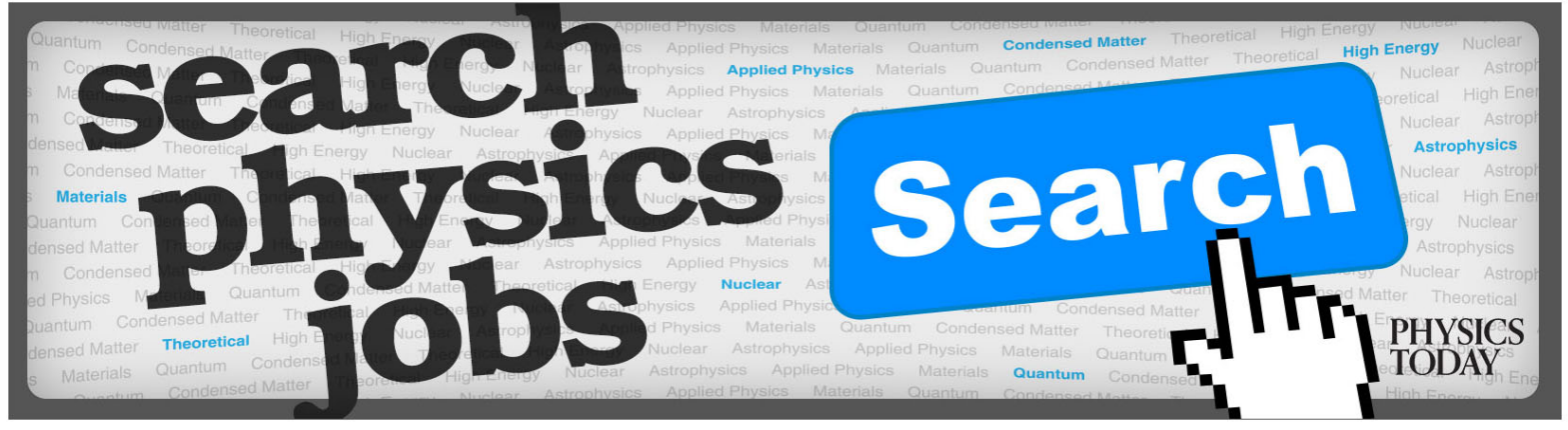




\title{
Multiplicity formulas for fundamental strings of representations of classical Lie algebras
}

\author{
Emilio A. Lauret ${ }^{1,2, a)}$ and Fiorela Rossi Bertone ${ }^{2, b)}$ \\ ${ }^{1}$ Institut für Mathematik, Humboldt Universität zu Berlin, Unter den Linden 6, \\ 10099 Berlin, Germany \\ ${ }^{2}$ CIEM-FaMAF (CONICET), Universidad Nacional de Córdoba, Medina Allende, \\ Ciudad Universitaria, 5000 Córdoba, Argentina
}

(Received 1 July 2017; accepted 7 November 2017; published online 27 November 2017)

\begin{abstract}
We call the p-fundamental string of a complex simple Lie algebra to the sequence of irreducible representations having highest weights of the form $k \omega_{1}+\omega_{p}$ for $k \geq 0$, where $\omega_{j}$ denotes the $j$ th fundamental weight of the associated root system. For a classical complex Lie algebra, we establish a closed explicit formula for the weight multiplicities of any representation in any $p$-fundamental string. Published by AIP Publishing. https://doi.org/10.1063/1.4993851
\end{abstract}

\section{INTRODUCTION}

Let $\mathfrak{g}$ be a complex semisimple Lie algebra. We fix a Cartan subalgebra $\mathfrak{h}$ of $\mathfrak{g}$. Let $\left(\pi, V_{\pi}\right)$ be a finite dimensional representation of $\mathfrak{g}$, that is, a homomorphism $\pi: \mathfrak{g} \rightarrow \mathfrak{g l}\left(V_{\pi}\right)$ with $V_{\pi}$ a complex vector space. An element $\mu \in \mathfrak{h}^{*}$ is called a weight of $\pi$ if

$$
V_{\pi}(\mu):=\left\{v \in V_{\pi}: \pi(X) v=\mu(X) v \text { for all } X \in \mathfrak{h}\right\} \neq 0 .
$$

The multiplicity of $\mu$ in the representation $\pi$, denoted by $m_{\pi}(\mu)$, is defined as $\operatorname{dim} V_{\pi}(\mu)$.

There are many formulas in the literature to compute $m_{\pi}(\mu)$ for arbitrary $\mathfrak{g}, \pi$, and $\mu$. The ones by Freudenthal ${ }^{12}$ and Kostant ${ }^{19}$ are very classical. More recent formulas were given by Lusztig, ${ }^{25}$ Littelmann, ${ }^{24}$ and Sahi. ${ }^{28}$ Although all of them are very elegant and powerful theoretical results, they may not be considered closed explicit expressions. Moreover, some of them are not adequate for computer implementation (cf. in Refs. 14 and 29).

Actually, it is not expected a closed formula in general. There should always be a sum over a symmetric group (whose cardinal grows quickly when the rank of $g$ does) or over partitions, or being recursive, or written in terms of combinatorial objects (e.g., Young diagrams like in Ref. 17), among other ways.

However, closed explicit expressions are possible for particular choices of $\mathfrak{g}$ and $\pi$. Obviously, this is the case for $\mathfrak{s I}(2, \mathbb{C})$ and $\pi$ any of its irreducible representations (see Sec. I.9 in Ref. 16). Furthermore, for a classical Lie algebra $\mathfrak{g}$, it is not difficult to give expressions for the weight multiplicities of the representations $\operatorname{Sym}^{k}\left(V_{\text {st }}\right)$ and $\bigwedge^{p}\left(V_{\text {st }}\right)$ and also for their irreducible components (see, for instance, Lemmas III.2, IV.3, and V.3 and Theorem VI.1; these formulas are probably well known but they are included here for completeness). Here, $V_{\text {st }}$ denotes the standard representation of $\mathfrak{g}$. A good example of a closed explicit formula in a non-trivial case was given by Cagliero and Tirao ${ }^{5}$ for $\mathfrak{s p}(2, \mathbb{C}) \simeq \mathfrak{s p}(5, \mathbb{C})$ and $\pi$ arbitrary.

In order to end the description of previous results in this large area, we name a few recent related results, though the list is far from being complete: Refs. 1, 2, 6-11, 26, and 30.

The main goal of this article is to show, for each classical complex Lie algebra $g$ of rank $n$, a closed explicit formula for the weight multiplicities of any irreducible representation of $\mathfrak{g}$ having highest weight $k \omega_{1}+\omega_{p}$, for any integers $k \geq 0$ and $1 \leq p \leq n$. Here, $\omega_{1}, \ldots, \omega_{n}$ denote the fundamental

\footnotetext{
a) Electronic mail: elauret@famaf.unc.edu.ar

b) Electronic mail: rossib@famaf.unc.edu.ar
} 
weights associated with the root system $\Sigma(\mathfrak{g}, \mathfrak{h})$. We call the $p$-fundamental string to the sequence of irreducible representations of $\mathfrak{g}$ with highest weights $k \omega_{1}+\omega_{p}$ for $k \geq 0$. We will write $\pi_{\lambda}$ for the irreducible representation of $\mathfrak{g}$ with highest weight $\lambda$.

For types $\mathrm{B}_{n}, \mathrm{C}_{n}$, or $\mathrm{D}_{n}$ [i.e., $\mathfrak{s v}(2 n+1, \mathbb{C}), \mathfrak{s p}(n, \mathbb{C})$, or $\mathfrak{s v}(2 n, \mathbb{C})$, respectively] an accessory representation $\pi_{k, p}$ is introduced to unify the approach (see Definition II.2). We have that $\pi_{k, p}$ and $\pi_{k \omega_{1}+\omega_{p}}$ coincide except for $p=n$ in type $\mathrm{B}_{n}$ and $p=n-1, n$ in type $\mathrm{D}_{n}$. The weight multiplicity formulas for $\pi_{k, p}$ are in Theorems III.1, IV.1, and V.1 for types $\mathrm{C}_{n}, \mathrm{D}_{n}$, and $\mathrm{B}_{n}$, respectively. Their proofs follow the same strategy (see Sec. II). The formulas for the remaining cases, namely, the (spin) representations $\pi_{k \omega_{1}+\omega_{n}}$ in type $\mathrm{B}_{n}$ and $\pi_{k \omega_{1}+\omega_{n-1}}, \pi_{k \omega_{1}+\omega_{n}}$ in type $\mathrm{D}_{n}$ can be found in Theorems IV.2 and V.4, respectively.

Given a weight $\mu=\sum_{j=1}^{n} a_{j} \varepsilon_{j}$ (see Notation II.1) of a classical Lie algebra $\mathfrak{g}$ of types $\mathrm{B}_{n}, \mathrm{C}_{n}$, or $\mathrm{D}_{n}$, we set

$$
\|\mu\|_{1}=\sum_{j=1}^{n}\left|a_{j}\right| \quad \text { and } \quad Z(\mu)=\#\left\{1 \leq j \leq n: a_{j}=0\right\} .
$$

We call $\|\mu\|_{1}$ the one-norm of $\mu$. The function $Z(\mu)$ counts the number of zero coordinates of $\mu$. It is not difficult to check that $m_{\pi_{k \omega_{1}}}(\mu)$ depends only on $\|\mu\|_{1}$ for a fixed $k \geq 0$. Moreover, it is known that $m_{\pi_{k, p}}(\mu)$ depends only on $\|\mu\|_{1}$ and $Z(\mu)$ for type $\mathrm{D}_{n}$ (see Lemma 3.3 in Ref. 23). This last property is extended to types $\mathrm{B}_{n}$ and $\mathrm{C}_{n}$ as a consequence of their multiplicity formulas.

Corollary I.1. For $\mathfrak{g}$, a classical Lie algebra of types $\mathrm{B}_{n}, \mathrm{C}_{n}$, or $\mathrm{D}_{n}$ and a weight $\mu=\sum_{i=1}^{n} a_{i} \varepsilon_{i}$, the multiplicity of $\mu$ in $\pi_{k, p}$ depends only on $\|\mu\|_{1}$ and $Z(\mu)$.

For $\mathfrak{g}=\mathfrak{s l}(n+1, \mathbb{C})$ (type $\left.\mathrm{A}_{n}\right)$, the multiplicity formula for a representation in a fundamental string is in Theorem VI.1. This case is simpler since it follows immediately from basic facts on Young diagrams. Although this formula should be well known, it is included for completeness.

Explicit expressions for the weight multiplicities of a representation in a fundamental string are required in several different areas. The interest of the authors on them comes from their application to spectral geometry. Actually, many multiplicity formulas have already been applied to determine the spectrum of Laplace and Dirac operators on certain locally homogeneous spaces. See Sec. VII for a detailed account of these applications.

It is important to note that all the weight multiplicity formulas obtained in this article have been checked with Sage $^{31}$ for many cases. This computer program uses the classical Freudenthal formula. Because of the simplicity of the expressions obtained in the main theorems, the computer takes usually a fraction of a second to calculate the result.

Throughout the article, we use the convention $\left(\begin{array}{l}b \\ a\end{array}\right)=0$ if $a<0$ or $b<a$.

The article is organized as follows. Section II explains the method to obtain $m_{\pi_{k, p}}(\mu)$ for types $\mathrm{B}_{n}, \mathrm{C}_{n}$, and $\mathrm{D}_{n}$. These cases are considered in Secs. V, III, and IV, respectively, and type $\mathrm{A}_{n}$ is in Sec. VI. In Sec. VII, we include some conclusions.

\section{STRATEGY}

In this section, we introduce the abstract method used to find the weight multiplicity formulas for the cases $\mathrm{B}_{n}, \mathrm{C}_{n}$, and $\mathrm{D}_{n}$. Throughout this section, $\mathfrak{g}$ denotes a classical complex Lie algebra of types $\mathrm{B}_{n}, \mathrm{C}_{n}$, and $\mathrm{D}_{n}$, namely $\mathfrak{s v}(2 n+1, \mathbb{C}), \mathfrak{s p}(n, \mathbb{C}), \mathfrak{s v}(2 n, \mathbb{C})$, for some $n \geq 2$. We first introduce some standard notation.

Notation II.1. We fix a Cartan subalgebra $\mathfrak{h}$ of $\mathfrak{g}$. Let $\left\{\varepsilon_{1}, \ldots, \varepsilon_{n}\right\}$ be the standard basis of $\mathfrak{h}^{*}$. Thus, the sets of simple roots $\Pi(\mathfrak{g}, \mathfrak{h})$ are given by $\left\{\varepsilon_{1}-\varepsilon_{2}, \ldots, \varepsilon_{n-1}-\varepsilon_{n}, \varepsilon_{n}\right\}$ for type $\mathrm{B}_{n}$, $\left\{\varepsilon_{1}-\varepsilon_{2}, \ldots, \varepsilon_{n-1}-\varepsilon_{n}, 2 \varepsilon_{n}\right\}$ for type $\mathrm{C}_{n}$, and $\left\{\varepsilon_{1}-\varepsilon_{2}, \ldots, \varepsilon_{n-1}-\varepsilon_{n}, \varepsilon_{n-1}+\varepsilon_{n}\right\}$ for type $\mathrm{D}_{n}$. A precise choice for $\mathfrak{h}$ and $\varepsilon_{j}$ will be indicated in each type.

We denote by $\Sigma(\mathfrak{g}, \mathfrak{h})$ the set of roots, by $\Sigma^{+}(\mathfrak{g}, \mathfrak{h})$ the set of positive roots, by $\omega_{1}, \ldots, \omega_{n}$ the fundamental weights, by $P(\mathfrak{g})$ the (integral) weight space of $\mathfrak{g}$, and by $P^{++}(\mathfrak{g})$ the set of dominant weights. 
Let $\mathfrak{g}_{0}$ be the compact real form of $\mathfrak{g}$ associated with $\Sigma(\mathfrak{g}, \mathfrak{h})$, let $G$ be the compact linear group with Lie algebra $g_{0}$ [e.g., $G=\mathrm{SO}(2 n)$ for type $\mathrm{D}_{n}$ in place of $\operatorname{spin}(2 n)$ ], and let $T$ be the maximal torus in $G$ corresponding to $\mathfrak{h}$, that is, the Lie algebra t of $T$ is a real subalgebra of $\mathfrak{h}$. Write $P(G)$ for the set of $G$-integral weights and $P^{++}(G)=P(G) \cap P^{++}(\mathfrak{g})$.

By the highest weight theorem, the irreducible representations of $g$ and $G$ are in correspondence with elements in $P^{++}(\mathfrak{g})$ and $P^{++}(G)$, respectively. For $\lambda$, an integral dominant weight, we denote by $\pi_{\lambda}$ the associated irreducible representation of $\mathfrak{g}$.

We recall that, under Notation II.1, the fundamental weights are

$$
\begin{aligned}
& \text { in type } \mathrm{B}_{n}, \quad \omega_{p}= \begin{cases}\varepsilon_{1}+\cdots+\varepsilon_{p} & \text { if } 1 \leq p \leq n-1, \\
\frac{1}{2}\left(\varepsilon_{1}+\cdots+\varepsilon_{n}\right) & \text { if } p=n,\end{cases} \\
& \text { in type } \mathrm{C}_{n}, \quad \omega_{p}=\varepsilon_{1}+\cdots+\varepsilon_{p} \quad \text { for every } 1 \leq p \leq n \text {, } \\
& \text { in type } \mathrm{D}_{n}, \quad \omega_{p}= \begin{cases}\varepsilon_{1}+\cdots+\varepsilon_{p} & \text { if } 1 \leq p \leq n-2, \\
\frac{1}{2}\left(\varepsilon_{1}+\cdots+\varepsilon_{n-1}-\varepsilon_{n}\right) & \text { if } p=n-1, \\
\frac{1}{2}\left(\varepsilon_{1}+\cdots+\varepsilon_{n-1}+\varepsilon_{n}\right) & \text { if } p=n .\end{cases}
\end{aligned}
$$

We set $\widetilde{\omega}_{p}=\varepsilon_{1}+\cdots+\varepsilon_{p}$ for any $1 \leq p \leq n$. Thus, $\widetilde{\omega}_{p}=\omega_{p}$ excepts for type $\mathrm{B}_{n}$ and $p=n$ when $\widetilde{\omega}_{n}=2 \omega_{n}$, and for type $\mathrm{D}_{n}$ and $p \in\{n-1, n\}$ when $\widetilde{\omega}_{n-1}=\omega_{n-1}+\omega_{n}$ and $\widetilde{\omega}_{n}=2 \omega_{n}$.

Definition II.2. Let $\mathfrak{g}$ be a classical Lie algebra of types $\mathrm{B}_{n}, \mathrm{C}_{n}$, or $\mathrm{D}_{n}$. For $k \geq 0$ and $1 \leq p \leq n$ integers, let us denote by $\pi_{k, p}$ the irreducible representation of $\mathfrak{g}$ with highest weight $k \omega_{1}+\widetilde{\omega}_{p}$, except for $p=n$ and type $\mathrm{D}_{n}$ when we set $\pi_{k, n}=\pi_{k \omega_{1}+2 \omega_{n-1}} \oplus \pi_{k \omega_{1}+2 \omega_{n}}$. By convention, we set $\pi_{k, 0}=0$ for $k \geq 0$.

Next we explain the procedure to determine the multiplicity formula for $\pi_{k, p}$.

Step 1: Obtain the decomposition in irreducible representations of

$$
\sigma_{k, p}:=\pi_{k \omega_{1}} \otimes \pi_{\widetilde{\omega}_{p}},
$$

and consequently, write $\pi_{k, p}$ in terms of representations of the form (2) in the virtual representation ring. Fortunately, this decomposition is already known and coincides for the types $\mathrm{B}_{n}, \mathrm{C}_{n}$, and $\mathrm{D}_{n}$; thus, the second requirement has also a uniform statement (see Lemma II.3).

Step 2: Obtain a formula for the weight multiplicities of the extreme cases $\pi_{k \omega_{1}}$ and $\pi_{\widetilde{\omega}_{p}}$. It will be useful to realize these representations inside $\operatorname{Sym}^{k}\left(V_{\pi_{\omega_{1}}}\right)$ and $\bigwedge^{p}\left(V_{\pi_{\omega_{1}}}\right)$, respectively. Note that $\pi_{\omega_{1}}$ is the standard representation.

Step 3: Obtain a closed expression for the weight multiplicities on $\sigma_{k, p}$. This is the hardest step. One has that (see, for instance, Exercise V.14 in Ref. 16)

$$
m_{\sigma_{k, p}}(\mu)=\sum_{\eta} m_{\pi_{k \omega_{1}}}(\mu-\eta) m_{\pi_{\widetilde{\omega}_{p}}}(\eta)
$$

where the sum is over the weights of $\pi_{\widetilde{\omega}_{p}}$. Then, the multiplicity formulas obtained in step 2 can be applied.

Step 4: Obtain the weight multiplicity formula for $\pi_{k, p}$. We will replace the formula obtained in step 3 into the formula obtained in step 1.

The following result works out step 1 .

Lemma II.3. Let $\mathfrak{g}$ be a classical Lie algebra of types $\mathrm{B}_{n}, \mathrm{C}_{n}$, or $\mathrm{D}_{n}$ and let $k \geq 0,1 \leq p \leq n$ integers. Then

$$
\sigma_{k, p}=\pi_{k \omega_{1}} \otimes \pi_{\widetilde{\omega}_{p}}=\pi_{k-1,1} \otimes \pi_{0, p} \simeq \pi_{k, p} \oplus \pi_{k-1, p+1} \oplus \pi_{k-2, p} \oplus \pi_{k-1, p-1} .
$$

Furthermore, in the virtual ring of representations, we have that

$$
\pi_{k, p}=\sum_{j=1}^{p}(-1)^{j-1} \sum_{i=0}^{j-1} \sigma_{k+j-2 i, p-j} .
$$


Proof. The decomposition (4) is proved by Koike and Terada ${ }^{18}$ [see Example (3) in p. 510], though their results are much more general and this particular case was probably already known.

We now show (5). The case $p=1$ is trivial. Indeed, the right hand side equals $\sigma_{k+1,0}=\pi_{k, 1}$ by definition. We assume that the formula is valid for values lower than or equal to $p$. By this assumption and (4), we have that

$$
\begin{aligned}
\pi_{k, p+1} & =\sigma_{k+1, p}-\pi_{k+1, p}-\pi_{k-1, p}-\pi_{k, p-1} \\
& =\sigma_{k+1, p}-\sum_{j=1}^{p}(-1)^{j-1} \sum_{i=0}^{j-1} \sigma_{k+1+j-2 i, p-j}-\sum_{j=1}^{p}(-1)^{j-1} \sum_{i=0}^{j-1} \sigma_{k-1+j-2 i, p-j}-\sum_{j=1}^{p-1}(-1)^{j-1} \sum_{i=0}^{j-1} \sigma_{k+j-2 i, p-1-j} .
\end{aligned}
$$

By making the change of variables $h=j+1$ in the last term, one gets

$$
\pi_{k, p+1}=\sigma_{k+1, p}-\sum_{j=1}^{p}(-1)^{j-1} \sum_{i=0}^{j-1} \sigma_{k+1+j-2 i, p-j}-\sigma_{k, p-1}-\sum_{j=2}^{p}(-1)^{j-1} \sigma_{k+1-j, p-j} .
$$

The rest of the proof is straightforward.

\section{TYPE C}

In this section, we consider the classical Lie algebra $\mathfrak{g}$ of type $\mathrm{C}_{n}$, that is, $\mathfrak{g}=\mathfrak{s p}(n, \mathbb{C})$. In this case, according to Notation II. $1, \widetilde{\omega}_{p}=\omega_{p}$ for every $p$, thus $\pi_{k \omega_{1}+\omega_{p}}=\pi_{k, p}$. The next theorem gives the explicit expression of $m_{\pi_{k, p}}(\mu)$ for any weight $\mu$. This expression depends on the terms $\|\mu\|_{1}$ and $Z(\mu)$, introduced in (1).

Theorem III.1. Let $\mathfrak{g}=\mathfrak{s p}(n, \mathbb{C})$ for some $n \geq 2$ and let $k \geq 0,1 \leq p \leq n$ integers. For $\mu \in P(\mathfrak{g})$, if $r(\mu):=\left(k+p-\|\mu\|_{1}\right) / 2$ is a non-negative integer, then

$$
\begin{aligned}
m_{\pi_{k, p}}(\mu)= & \sum_{j=1}^{p}(-1)^{j-1} \sum_{t=0}^{\left\lfloor\frac{p-j}{2}\right\rfloor} \frac{n-p+j+1}{n-p+j+t+1}\left(\begin{array}{c}
n-p+j+2 t \\
t
\end{array}\right) \sum_{\beta=0}^{p-j-2 t} 2^{p-j-2 t-\beta}\left(\begin{array}{c}
n-Z(\mu) \\
\beta
\end{array}\right)\left(\begin{array}{c}
Z(\mu) \\
p-j-2 t-\beta
\end{array}\right) \\
& \sum_{\alpha=0}^{\beta}\left(\begin{array}{l}
\beta \\
\alpha
\end{array}\right) \sum_{i=0}^{j-1}\left(\begin{array}{c}
r(\mu)-i-p+\alpha+t+j+n-1 \\
n-1
\end{array}\right),
\end{aligned}
$$

and $m_{\pi_{k, p}}(\mu)=0$ otherwise.

The rest of this section is devoted to prove this formula following the procedure described in Sec. II. We first set the notation for this case. Here $G=\operatorname{Sp}(n, \mathbb{C}) \cap \mathrm{U}(2 n)$ where $\operatorname{Sp}(n, \mathbb{C})$ $=\left\{g \in \mathrm{SL}(2 n, \mathbb{C}): g^{t} J_{n} g=J_{n}:=\left(\begin{array}{cc}0 & \mathrm{Id}_{n} \\ -\mathrm{Id}_{n} & 0\end{array}\right)\right\}, \mathfrak{g}_{0}=\mathfrak{s p}(n, \mathbb{C}) \cap \mathfrak{u}(2 n)$,

$$
\begin{aligned}
T & =\left\{\operatorname{diag}\left(e^{\mathrm{i} \theta_{1}}, \ldots, e^{\mathrm{i} \theta_{n}}, e^{-\mathrm{i} \theta_{1}}, \ldots, e^{-\mathrm{i} \theta_{n}}\right): \theta_{i} \in \mathbb{R} \forall i\right\}, \\
\mathfrak{h} & =\left\{\operatorname{diag}\left(\theta_{1}, \ldots, \theta_{n},-\theta_{1}, \ldots,-\theta_{n}\right): \theta_{i} \in \mathbb{C} \forall i\right\},
\end{aligned}
$$

$\varepsilon_{i}\left(\operatorname{diag}\left(\theta_{1}, \ldots, \theta_{n},-\theta_{1}, \ldots,-\theta_{n}\right)\right)=\theta_{i}$ for each $1 \leq i \leq n, \Sigma^{+}(\mathfrak{g}, \mathfrak{h})=\left\{\varepsilon_{i} \pm \varepsilon_{j}: 1 \leq i<j \leq n\right\} \cup\left\{2 \varepsilon_{i}\right.$ : $1 \leq i \leq n\}$, and

$$
\begin{aligned}
P(\mathfrak{g}) & =P(G)=\mathbb{Z} \varepsilon_{1} \oplus \cdots \oplus \mathbb{Z} \varepsilon_{n}, \\
P^{++}(\mathfrak{g}) & =P^{++}(G)=\left\{\sum_{i} a_{i} \varepsilon_{i} \in P(\mathfrak{g}): a_{1} \geq a_{2} \geq \cdots \geq a_{n} \geq 0\right\} .
\end{aligned}
$$

The following well-known identities (see, for instance, Sec. 17.2 in Ref. 13) will be useful to show step 2,

$$
\pi_{k \omega_{1}}=\pi_{k \varepsilon_{1}} \simeq \operatorname{Sym}^{k}\left(\mathbb{C}^{2 n}\right), \quad \bigwedge^{p}\left(\mathbb{C}^{2 n}\right) \simeq \pi_{\omega_{p}} \oplus \bigwedge^{p-2}\left(\mathbb{C}^{2 n}\right),
$$

for any integers $k \geq 0$ and $1 \leq p \leq n$. Here, $\mathbb{C}^{2 n}$ denotes the standard representation of $\mathfrak{g}=\mathfrak{s p}(2 n, \mathbb{C})$. Since $G=\operatorname{Sp}(n)$ is simply connected, $\pi_{\lambda}$ descends to a representation of $G$ for any $\lambda \in P^{++}(\mathrm{g})$. In what follows we will work with representations of $G$ for simplicity. Thus, $m_{\pi}(\mu)=\operatorname{dim}\left\{v \in V_{\pi}\right.$ : $\left.\pi(\exp X) v=e^{\mu(X)} v \quad \forall X \in \mathrm{t}\right\}$. 
Lemma III.2. Let $n \geq 2, \mathfrak{g}=\mathfrak{s p}(n, \mathbb{C}), k \geq 0,1 \leq p \leq n$, and $\mu=\sum_{j=1}^{n} a_{j} \varepsilon_{j} \in P(\mathfrak{g})$. Then,

$$
\begin{aligned}
& m_{\pi_{k \omega_{1}}}(\mu)=m_{\pi_{k \varepsilon_{1}}}(\mu)= \begin{cases}\left(\begin{array}{c}
r(\mu)+n-1 \\
n-1
\end{array}\right) & \text { if } r(\mu):=\frac{k-\|\mu\|_{1}}{2} \in \mathbb{N}_{0}, \\
0 & \text { otherwise, }\end{cases} \\
& m_{\pi_{\omega_{p}}}(\mu)= \begin{cases}\frac{n-p+1}{n-p+r(\mu)+1}\left(\begin{array}{c}
n-p+2 r(\mu) \\
r(\mu)
\end{array}\right) \text { if } r(\mu):=\frac{p-\|\mu\|_{1}}{2} \in \mathbb{N}_{0} \text { and }\left|a_{j}\right| \leq 1 \forall j, \\
0 & \text { otherwise. }\end{cases}
\end{aligned}
$$

Proof. By (8), $\pi_{k \varepsilon_{1}}$ is realized in the space of homogeneous polynomials $\mathcal{P}_{k} \simeq \operatorname{Sym}^{k}\left(\mathbb{C}^{2 n}\right)$ of degree $k$ in the variables $x_{1}, \ldots, x_{2 n}$. The action of $g \in G$ on $f(x) \in \mathcal{P}_{k}$ is given by $\left(\pi_{k \varepsilon_{1}}(g) \cdot f\right)(x)$ $=f\left(g^{-1} x\right)$, where $x$ denotes the column vector $\left(x_{1}, \ldots, x_{2 n}\right)^{t}$.

The monomials $x_{1}^{k_{1}} \ldots x_{n}^{k_{n}} x_{n+1}^{l_{1}} \ldots x_{2 n}^{l_{n}}$ with $k_{1}, \ldots, k_{n}, l_{1}, \ldots, l_{n}$ non-negative integers satisfying that $\sum_{j=1}^{n} k_{j}+l_{j}=k$ form a basis of $\mathcal{P}_{k}$ given by weight vectors. Indeed, one can check that the action of the element $h=\operatorname{diag}\left(e^{\mathrm{i} \theta_{1}}, \ldots, e^{\mathrm{i} \theta_{n}}, e^{-\mathrm{i} \theta_{1}}, \ldots, e^{-\mathrm{i} \theta_{n}}\right) \in T$ on the monomial $x_{1}^{k_{1}} \ldots x_{n}^{k_{n}} x_{n+1}^{l_{1}} \ldots x_{2 n}^{l_{n}}$ is given by the multiplication by $e^{i \sum_{j=1}^{n} \theta_{j}\left(k_{j}-l_{j}\right)}$. Hence, the polynomial $x_{1}^{k_{1}} \ldots x_{n}^{k_{n}} x_{n+1}^{l_{1}} \ldots x_{2 n}^{l_{n}}$ is a weight vector of weight $\mu=\sum_{j=1}^{n}\left(k_{j}-l_{j}\right) \varepsilon_{j}$.

Consequently, the multiplicity of a weight $\mu=\sum_{j=1}^{n} a_{j} \varepsilon_{j} \in \mathcal{P}(\mathfrak{g})$ in $\mathcal{P}_{k}$ is the number of different tuples $\left(k_{1}, \ldots, k_{n}, l_{1}, \ldots, l_{n}\right) \in \mathbb{N}_{0}^{2 n}$ satisfying $\sum_{j=1}^{n}\left(k_{j}+l_{j}\right)=k$ and $a_{j}=k_{j}-l_{j}$ for all $j$. For such a tuple, we note that $k-\|\mu\|_{1}=k-\sum_{i=1}^{n}\left|a_{i}\right|=2 \sum_{i=1}^{n} \min \left(k_{i}, l_{i}\right)$. It follows that $\mu$ is a weight of $\mathcal{P}_{k}$ if and only if $k-\|\mu\|_{1}=2 r$ with $r$ a non-negative integer. Moreover, its multiplicity is the number of different ways one can write $r$ as an ordered sum of $n$ non-negative integers, which equals $\left(\begin{array}{c}r+n-1 \\ n-1\end{array}\right)$. This implies (9).

For $(10)$, we consider the representation $\bigwedge^{p}\left(\mathbb{C}^{2 n}\right)$. The action of $G$ on $\bigwedge^{p}\left(\mathbb{C}^{2 n}\right)$ is given by $g \cdot v_{1}$ $\wedge \cdots \wedge v_{p}=\left(g v_{1}\right) \wedge \cdots \wedge\left(g v_{p}\right)$, where $g v$ stands for the matrix multiplication between $g \in G \subset \mathrm{GL}(2 n, \mathbb{C})$ and the column vector $v \in \mathbb{C}^{2 n}$.

Let $\left\{e_{1}, \ldots, e_{2 n}\right\}$ denote the canonical basis of $\mathbb{C}^{2 n}$. For $I=\left\{i_{1}, \ldots, i_{p}\right\}$ with $1 \leq i_{1}<\cdots<i_{p} \leq 2 n$, we write $w_{I}=e_{i_{1}} \wedge \cdots \wedge e_{i_{p}}$. Clearly, the set of $w_{I}$ for all choices of $I$ is a basis of $\wedge^{p}\left(\mathbb{C}^{2 n}\right)$. Since $h$ $=\operatorname{diag}\left(e^{\mathrm{i} \theta_{1}}, \ldots, e^{\mathrm{i} \theta_{n}}, e^{-\mathrm{i} \theta_{1}}, \ldots, e^{-\mathrm{i} \theta_{n}}\right) \in T$ satisfies $h e_{j}=e^{\mathrm{i} \theta_{j}} e_{j}$ and $h e_{j+n}=e^{-\mathrm{i} \theta_{j}} e_{j+n}$ for all $1 \leq j \leq n$, we see that $w_{I}$ is a weight vector of weight $\mu=\sum_{j=1}^{n} a_{j} \varepsilon_{j}$ where

$$
a_{j}= \begin{cases}1 & \text { if } j \in I \text { and } j+n \notin I, \\ -1 & \text { if } j \notin I \text { and } j+n \in I, \\ 0 & \text { if } j, j+n \in I \text { or } j, j+n \notin I .\end{cases}
$$

Thus, an arbitrary element $\mu=\sum_{j} a_{j} \varepsilon_{j} \in P(\mathfrak{g})$ is a weight of $\bigwedge^{p}\left(\mathbb{C}^{2 n}\right)$ if and only if $\left|a_{j}\right| \leq 1$ for all $j$ and $p-\|\mu\|_{1}=2 r$ for some non-negative integer $r$.

It remains to determine the multiplicity in $\bigwedge^{p}\left(\mathbb{C}^{2 n}\right)$ of a weight $\mu=\sum_{j=1}^{n} a_{j} \varepsilon_{j} \in P(\mathfrak{g})$ satisfying $\left|a_{j}\right| \leq 1$ for all $j$ and $r:=\frac{p-\|\mu\|_{1}}{2} \in \mathbb{N}_{0}$. Let $I_{\mu}=\left\{i: 1 \leq i \leq n, a_{i}=1\right\} \cup\left\{i: n+1 \leq i \leq 2 n, a_{i-n}\right.$ $=-1\}$. The set $I_{\mu}$ has $p-2 r$ elements. For $I=\left\{i_{1}, \ldots, i_{p}\right\}$ with $1 \leq i_{1}<\cdots<i_{p} \leq 2 n$, it is a simple matter to check that $w_{I}$ is a weight vector with weight $\mu$ if and only if $I$ has $p$ elements, $I_{\mu} \subset I$ and $I$ has the property that $j \in I \backslash I_{\mu} \Leftrightarrow j+n \in I \backslash I \mu$ for $1 \leq j \leq n$. One can see that there are $\left(\begin{array}{c}n-p+2 r \\ r\end{array}\right)$ choices for I. Hence $m_{\wedge^{p}\left(\mathbb{C}^{2 n)}\right.}(\mu)=\left(\begin{array}{c}n-p+2 r \\ r\end{array}\right)$. From (8), we conclude that $m_{\pi_{\omega_{p}}}(\mu)=m_{\wedge^{p}\left(\mathbb{C}^{2 n}\right)}(\mu)-m_{\wedge^{p-2}\left(\mathbb{C}^{2 n}\right)}(\mu)$ $=\left(\begin{array}{c}n-p+2 r \\ r\end{array}\right)-\left(\begin{array}{c}n-p+2+2 r \\ r\end{array}\right)$ and (10) is proved.

We next consider step 3, namely, a multiplicity formula for $\sigma_{k, p}$.

Lemma III.3. Let $n \geq 2, \mathfrak{g}=\mathfrak{s p}(n, \mathbb{C}), k \geq 0,1 \leq p<n$, and $\mu \in P(\mathfrak{g})$. If $r(\mu):=\left(k+p-\|\mu\|_{1}\right) / 2$ is a non-negative integer, then 


$$
\begin{aligned}
m_{\sigma_{k, p}}(\mu)= & \sum_{t=0}^{\lfloor p / 2\rfloor} \frac{n-p+1}{n-p+t+1}\left(\begin{array}{c}
n-p+2 t \\
t
\end{array}\right) \sum_{\beta=0}^{p-2 t} 2^{p-2 t-\beta}\left(\begin{array}{c}
n-Z(\mu) \\
\beta
\end{array}\right)\left(\begin{array}{c}
Z(\mu) \\
p-2 t-\beta
\end{array}\right) \\
& \sum_{\alpha=0}^{\beta}\left(\begin{array}{c}
\beta \\
\alpha
\end{array}\right)\left(\begin{array}{c}
r(\mu)-p+\alpha+t+n-1 \\
n-1
\end{array}\right),
\end{aligned}
$$

and $m_{\sigma_{k, p}}(\mu)=0$ otherwise.

Proof. Write $r=r(\mu)$ and $\ell=Z(\mu)$. We may assume that $\mu$ is dominant, thus $\mu=\sum_{j=1}^{n-\ell} a_{j} \varepsilon_{j}$ with $a_{1} \geq \cdots \geq a_{n-\ell}>0$ since it has $\ell$ zero-coordinates. In order to use (3), by Lemma III.2, we write the set of weights of $\pi_{\omega_{p}}$ as

$$
\mathcal{P}\left(\pi_{\omega_{p}}\right):=\bigcup_{t=0}^{\lfloor p / 2\rfloor} \bigcup_{\beta=0}^{p-2 t} \bigcup_{\alpha=0}^{\beta} \mathcal{P}_{t, \beta, \alpha}^{(p)}
$$

where

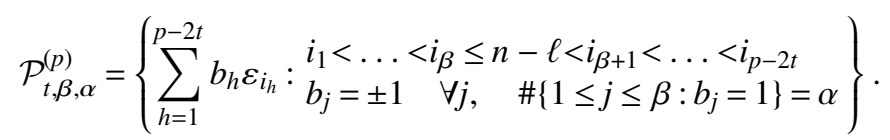

A weight $\eta \in \mathcal{P}_{t, \beta, \alpha}^{(p)}$ has all entries in $\{0, \pm 1\}$ and satisfies $\|\eta\|_{1}=p-2 t$, thus $m_{\pi_{\omega_{p}}}(\eta)$ $=\frac{n-p+1}{n-p+t+1}\left(\begin{array}{c}n-p+2 t \\ t\end{array}\right)$ by (10). It is a simple matter to check that

$$
\# \mathcal{P}_{t, \beta, \alpha}^{(p)}=2^{p-2 t-\beta}\left(\begin{array}{c}
n-\ell \\
\beta
\end{array}\right)\left(\begin{array}{c}
\beta \\
\alpha
\end{array}\right)\left(\begin{array}{c}
\ell \\
p-2 t-\beta
\end{array}\right) .
$$

From (3), since the triple union above is disjoint, we obtain that

$$
m_{\sigma_{k, p}}(\mu)=\sum_{t=0}^{\lfloor p / 2\rfloor} \sum_{\beta=0}^{p-2 t} \sum_{\alpha=0}^{\beta} \sum_{\eta \in \mathcal{P}_{t, \beta, \alpha}^{(p)}} m_{\pi_{k \varepsilon_{1}}}(\mu-\eta) m_{\pi_{\omega_{p}}}(\eta) .
$$

One has that $\|\mu-\eta\|_{1}=(k+p-2 r)+(\beta-\alpha)-\alpha+(p-2 t-\beta)=k-2(r+t+\alpha-p)$ for every $\eta \in \mathcal{P}_{t, \beta, \alpha}^{(p)}$. If $r \notin \mathbb{N}_{0}$, (9) forces $m_{\pi_{k \varepsilon_{1}}}(\mu-\eta)=0$ for all $\eta \in \mathcal{P}_{t, \beta, \alpha}^{(p)}$, consequently $m_{\sigma_{k, p}}(\mu)=0$. Otherwise,

$$
m_{\sigma_{k, p}}(\mu)=\sum_{t=0}^{\lfloor p / 2\rfloor} \sum_{\beta=0}^{p-2 t} \sum_{\alpha=0}^{\beta}\left(\begin{array}{c}
r+t+\alpha-p+n-1 \\
n-1
\end{array}\right) \frac{n-p+1}{n-p+t+1}\left(\begin{array}{c}
n-p+2 t \\
t
\end{array}\right) \# \mathcal{P}_{t, \beta, \alpha}^{(p)}
$$

by Lemma III.2. The proof is complete by (13).

Theorem III.1 follows by replacing the multiplicity formula given in Lemma III.3 into (5).

\section{TYPE D}

We now consider type $\mathrm{D}_{n}$, that is, $\mathfrak{g}=\mathfrak{s p}(2 n, \mathbb{C})$ and $G=\mathrm{SO}(2 n)$. We assume that $n \geq 2$, so the non-simple case $\mathfrak{g}=\mathfrak{s \mathfrak { D }}(4, \mathbb{C}) \simeq \mathfrak{s l}(2, \mathbb{C}) \oplus \mathfrak{s l}(2, \mathbb{C})$ is also considered.

Since $G$ is not simply connected and has a fundamental group of order 2, the lattice of $G$-integral weights $P(G)$ is strictly included with index 2 in the weight space $P(\mathfrak{g})$. Consequently, a dominant weight $\lambda$ in $P(\mathfrak{g}) \backslash P(G)$ corresponds to a representation $\pi_{\lambda}$ of $\operatorname{spin}(2 n)$, which does not descend to a representation of $G=\mathrm{SO}(2 n)$.

In this case, for all $k \geq 0$ and $1 \leq p \leq n-2$, we have that

$$
\pi_{k, p}=\pi_{k \omega_{1}+\omega_{p}}, \quad \pi_{k, n-1}=\pi_{k \omega_{1}+\omega_{n-1}+\omega_{n}}, \quad \pi_{k, n}=\pi_{k \omega_{1}+2 \omega_{n-1}} \oplus \pi_{k \omega_{1}+2 \omega_{n}} .
$$

Each of them descends to a representation of $G$ and its multiplicity formula is established in Theorem IV.1. The remaining cases $\pi_{k \omega_{1}+\omega_{n}-1}$ and $\pi_{k \omega_{1}+\omega_{n}}$ are spin representations. Their multiplicity formulas were obtained in Ref. 4 (Lemma 4.2) and are stated in Theorem IV.2. 
Theorem IV.1. Let $\mathfrak{g}=\mathfrak{s o}(2 n, \mathbb{C})$ and $G=\operatorname{SO}(2 n)$ for some $n \geq 2$ and let $k \geq 0,1 \leq p \leq n$ integers. For $\mu \in P(G)$, if $r(\mu):=\left(k+p-\|\mu\|_{1}\right) / 2$ is a non-negative integer, then

$$
\begin{aligned}
m_{\pi_{k, p}}(\mu)= & \sum_{j=1}^{p}(-1)^{j-1} \sum_{t=0}^{\left\lfloor\frac{p-j}{2}\right\rfloor}\left(\begin{array}{c}
n-p+j+2 t \\
t
\end{array}\right) \sum_{\beta=0}^{p-j-2 t} 2^{p-j-2 t-\beta}\left(\begin{array}{c}
n-Z(\mu) \\
\beta
\end{array}\right)\left(\begin{array}{c}
Z(\mu) \\
p-j-2 t-\beta
\end{array}\right) \\
& \sum_{\alpha=0}^{\beta}\left(\begin{array}{l}
\beta \\
\alpha
\end{array}\right) \sum_{i=0}^{j-1}\left(\begin{array}{c}
r(\mu)-i-p+\alpha+t+j+n-2 \\
n-2
\end{array}\right),
\end{aligned}
$$

and $m_{\pi_{k, p}}(\mu)=0$ otherwise. Furthermore, $m_{\pi_{k, p}}(\mu)=0$ for every $\mu \in P(\mathfrak{g}) \backslash P(G)$.

Theorem IV.2. Let $\mathfrak{g}=\mathfrak{s p}(2 n, \mathbb{C})$ and $G=\mathrm{SO}(2 n)$ for some $n \geq 2$ and let $k \geq 0$ an integer. Let $\mu \in P(\mathfrak{g}) \backslash P(G)$. Write $r(\mu)=k+\frac{n}{2}-\|\mu\|_{1}$, then

$$
\begin{aligned}
m_{\pi_{k \omega_{1}+\omega_{n}}}(\mu) & = \begin{cases}\left(\begin{array}{c}
r(\mu)+n-2 \\
n-2
\end{array}\right) & \text { if } r(\mu) \geq 0 \text { and } \operatorname{neg}(\mu) \equiv r(\mu) \quad(\bmod 2), \\
0 & \text { otherwise, }\end{cases} \\
m_{\pi_{k \omega_{1}+\omega_{n-1}}}(\mu) & = \begin{cases}\left(\begin{array}{c}
r(\mu)+n-2 \\
n-2
\end{array}\right) & \text { if } r(\mu) \geq 0 \text { and } \operatorname{neg}(\mu) \equiv r(\mu)+1 \quad(\bmod 2), \\
0 & \text { otherwise, }\end{cases}
\end{aligned}
$$

where neg $(\mu)$ stands for the number of negative entries of $\mu$. Furthermore, $m_{\pi_{k \omega_{1}+\omega_{n-1}}}(\mu)=m_{\pi_{k \omega_{1}+\omega_{n}}}(\mu)$ $=0$ for every $\mu \in P(G)$.

The proof of Theorem IV.1 will follow the steps from Sec. II. Let us first set the elements introduced in Notation II.1. Define $\mathfrak{h}=\left\{\operatorname{diag}\left(\left[\begin{array}{cc}0 & \theta_{1} \\ -\theta_{1} & 0\end{array}\right], \ldots,\left[\begin{array}{cc}0 & \theta_{n} \\ -\theta_{n} & 0\end{array}\right]\right): \theta_{i} \in \mathbb{C} \forall i\right\}$ and $\varepsilon_{i}\left(\operatorname{diag}\left(\left[\begin{array}{cc}0 & \theta_{1} \\ -\theta_{1} & 0\end{array}\right], \ldots,\left[\begin{array}{cc}0 & \theta_{n} \\ -\theta_{n} & 0\end{array}\right]\right)\right)=\theta_{i}$ for each $1 \leq i \leq n$. Thus $\Sigma^{+}(\mathfrak{g}, \mathfrak{h})=\left\{\varepsilon_{i} \pm \varepsilon_{j}: i<j\right\}$,

$$
\begin{aligned}
P(\mathfrak{g}) & =\left\{\sum_{i} a_{i} \varepsilon_{i}: a_{i} \in \mathbb{Z} \forall i, \text { or } a_{i}-1 / 2 \in \mathbb{Z} \forall i\right\}, & P(G) & =\mathbb{Z} \varepsilon_{1} \oplus \cdots \oplus \mathbb{Z} \varepsilon_{n}, \\
P^{++}(\mathfrak{g}) & =\left\{\sum_{i} a_{i} \varepsilon_{i} \in P(\mathfrak{g}): a_{1} \geq \cdots \geq a_{n-1} \geq\left|a_{n}\right|\right\}, & P^{++}(G) & =P^{++}(\mathfrak{g}) \cap P(G) .
\end{aligned}
$$

It is now clear that $P(G)$ has index 2 in $P(\mathfrak{g})$.

The multiplicity formulas in type $\mathrm{D}_{n}$ for the extreme representations in step 2 are already determined. A proof can be found in Ref. 23 (Lemma 3.2).

Lemma IV.3. Let $n \geq 2, \mathfrak{g}=\mathfrak{s} \mathfrak{D}(2 n, \mathbb{C}), G=\mathrm{SO}(2 n), k \geq 0$, and $1 \leq p \leq n$. For $\mu=\sum_{j=1}^{n} a_{j} \varepsilon_{j} \in P(G)$, we have that

$$
\begin{aligned}
& m_{\pi_{k \omega_{1}}}(\mu)=m_{\pi_{k \varepsilon_{1}}}(\mu)= \begin{cases}\left(\begin{array}{c}
r(\mu)+n-2 \\
n-2
\end{array}\right) & \text { if } r(\mu):=\frac{k-\|\mu\|_{1}}{2} \in \mathbb{N}_{0}, \\
0 & \text { otherwise, }\end{cases} \\
& m_{\pi_{\tilde{\omega}_{p}}}(\mu)=\left\{\begin{array}{cc}
\left(\begin{array}{c}
n-p+2 r(\mu) \\
r(\mu)
\end{array}\right) & \text { if } r(\mu):=\frac{p-\|\mu\|_{1}}{2} \in \mathbb{N}_{0} \text { and }\left|a_{j}\right| \leq 1 \forall j, \\
0 & \text { otherwise. }
\end{array}\right.
\end{aligned}
$$

Lemma IV.4. Let $n \geq 2, \mathfrak{g}=\mathfrak{s v}(2 n, \mathbb{C}), G=\mathrm{SO}(2 n), k \geq 0,1 \leq p \leq n-1$, and $\mu \in P(G)$. Write $r(\mu)=\left(k+p-\|\mu\|_{1}\right) / 2$. If $r(\mu)$ is a non-negative integer, then

$$
m_{\sigma_{k, p}}(\mu)=\sum_{t=0}^{\lfloor p / 2\rfloor}\left(\begin{array}{c}
n-p+2 t \\
t
\end{array}\right) \sum_{\beta=0}^{p-2 t} 2^{p-2 t-\beta}\left(\begin{array}{c}
n-Z(\mu) \\
\beta
\end{array}\right)\left(\begin{array}{c}
Z(\mu) \\
p-2 t-\beta
\end{array}\right) \sum_{\alpha=0}^{\beta}\left(\begin{array}{l}
\beta \\
\alpha
\end{array}\right)\left(\begin{array}{c}
r(\mu)-p+\alpha+t+n-2 \\
n-2
\end{array}\right),
$$

and $m_{\sigma_{k, p}}(\mu)=0$ otherwise.

Proof. We will omit several details in the rest of the proof since it is very similar to the one of Lemma III.3. Write $r=\left(k+p-\|\mu\|_{1}\right) / 2$ and $\ell=Z(\mu)$. We assume that $\mu$ is dominant. Lemma IV.3 implies that the set of weights of $\pi_{\widetilde{\omega}_{p}}$ is $\mathcal{P}\left(\pi_{\widetilde{\omega}_{p}}\right):=\bigcup_{t=0}^{\lfloor p / 2\rfloor} \bigcup_{\beta=0}^{p-2 t} \bigcup_{\alpha=0}^{\beta} \mathcal{P}_{t, \beta, \alpha}^{(p)}$, with $\mathcal{P}_{t, \beta, \alpha}^{(p)}$ as in (12). 
One has that $\|\mu-\eta\|_{1}=k-2(r+t+\alpha-p)$ for any $\eta \in \mathcal{P}_{t, \beta, \alpha}^{(p)}$. Hence, (3) and Lemma IV.3 imply $m_{\sigma_{k, p}}(\mu)=0$ if $r \notin \mathbb{N}_{0}$ and

$$
m_{\sigma_{k, p}}(\mu)=\sum_{t=0}^{\lfloor p / 2\rfloor} \sum_{\beta=0}^{p-2 t} \sum_{\alpha=0}^{\beta}\left(\begin{array}{c}
r+t+\alpha-p+n-2 \\
n-2
\end{array}\right)\left(\begin{array}{c}
n-p+2 t \\
t
\end{array}\right) \# \mathcal{P}_{t, \beta, \alpha}^{(p)}
$$

otherwise. The proof follows by (13).

Theorem IV.1 then follows by substituting in (5) the multiplicity formula in Lemma IV.4.

Remark IV.5. By Definition II.2, $\pi_{k, n}$ in type $\mathrm{D}_{n}$ is the only case where $\pi_{k, p}$ is not irreducible. We have that $\pi_{k, n}=\pi_{k \omega_{1}+\widetilde{\omega}_{n}} \oplus \pi_{k \omega_{1}+\widetilde{\omega}_{n}-2 \varepsilon_{n}}=\pi_{k \omega_{1}+2 \omega_{n-1}} \oplus \pi_{k \omega_{1}+2 \omega_{n}}$ for every $k \geq 0$. One can obtain the corresponding multiplicity formula for each of these irreducible constituents from Theorem IV.1 by proving the following facts. If $\mu \in P(G)$ satisfies $\|\mu\|_{1}=k+n$, then $m_{\pi_{k \omega_{1}+2 \omega_{n}}}(\mu)=m_{\pi_{k, n}}(\mu)$ and $m_{\pi_{k \omega_{1}+2 \omega_{n-1}}}(\mu)=0$ or $m_{\pi_{k \omega_{1}+2 \omega_{n}}}(\mu)=0$ and $m_{\pi_{k \omega_{1}+2 \omega_{n-1}}}(\mu)=m_{\pi_{k, n}}(\mu)$ and accordingly $\mu$ has an even or odd number of negative entries, respectively. Furthermore, if $\mu \in P(G)$ satisfies $\|\mu\|_{1}<k+n$, then $m_{\pi_{k \omega_{1}+2 \omega_{n}}}(\mu)=m_{\pi_{k \omega_{1}+2 \omega_{n-1}}}(\mu)=m_{\pi_{k, n}}(\mu) / 2$.

\section{TYPE B}

We now consider $\mathfrak{g}=\mathfrak{s p}(2 n+1, \mathbb{C})$ and $G=\mathrm{SO}(2 n+1)$, so $\mathfrak{g}$ is of type $\mathrm{B}_{n}$. The same observation in the beginning of Sec. IV is valid in this case. Namely, a weight in $P^{++}(\mathfrak{g}) \backslash P^{++}(G)$ induces an irreducible representation of $\operatorname{spin}(2 n+1)$ which does not descend to $G$.

For any $k \geq 0$ and $1 \leq p \leq n-1$, we have that

$$
\pi_{k, p}=\pi_{k \omega_{1}+\omega_{p}}, \quad \pi_{k, n}=\pi_{k \omega_{1}+2 \omega_{n}} .
$$

All of them descend to representations of $G$. The corresponding multiplicity formula is in Theorem V.1 and the remaining case, $\pi_{k \omega_{1}+\omega_{n}}$ for $k \geq 0$, is considered in Theorem V.4.

Theorem V.1. Let $\mathfrak{g}=\mathfrak{s o}(2 n+1), G=\mathrm{SO}(2 n+1)$ for some $n \geq 2$ and let $k \geq 0,1 \leq p \leq n$ integers. For $\mu \in P(G)$, write $r(\mu)=k+p-\|\mu\|_{1}$, then

$$
\begin{aligned}
& m_{\pi_{k, p}}(\mu)= \sum_{j=1}^{p}(-1)^{j-1} \sum_{t=0}^{\left\lfloor\frac{p-j}{2}\right\rfloor}\left(\begin{array}{c}
n-p+j+2 t \\
t
\end{array}\right) \sum_{\beta=0}^{p-j-2 t} 2^{p-j-2 t-\beta}\left(\begin{array}{c}
n-Z(\mu) \\
\beta
\end{array}\right)\left(\begin{array}{c}
Z(\mu) \\
p-j-2 t-\beta
\end{array}\right) \\
& \sum_{\alpha=0}^{\beta}\left(\begin{array}{l}
\beta \\
\alpha
\end{array}\right) \sum_{i=0}^{j-1}\left(\begin{array}{c}
\left.\frac{r(\mu)}{2}\right\rfloor-i-p+j+\alpha+t+n-1 \\
n-1
\end{array}\right) \\
&+ \sum_{j=1}^{p-1}(-1)^{j-1} \sum_{t=0}^{\left\lfloor\frac{p-j-1}{2}\right\rfloor}\left(\begin{array}{c}
n-p+j+2 t+1 \\
t
\end{array}\right) \sum_{\beta=0}^{p-j-2 t-1} 2^{p-j-2 t-\beta-1}\left(\begin{array}{c}
n-Z(\mu) \\
\beta
\end{array}\right)\left(\begin{array}{c}
Z(\mu) \\
p-j-2 t-\beta-1
\end{array}\right) \\
& \sum_{\alpha=0}^{\beta}\left(\begin{array}{l}
\beta \\
\alpha
\end{array}\right) \sum_{i=0}^{j-1}\left(\begin{array}{c}
\left.\frac{r(\mu)+1}{2}\right\rfloor-i-p+j+\alpha+t+n-1 \\
n-1
\end{array}\right) .
\end{aligned}
$$

Furthermore, $m_{\pi_{k, p}}(\mu)=0$ for all $\mu \in P(\mathfrak{g}) \backslash P(G)$.

Remark V.2. Notice that, in Theorem V.1, $m_{\pi_{k, p}}(\mu)=0$ if $r(\mu)<0$ because of the convention $\left(\begin{array}{l}b \\ a\end{array}\right)=0$ if $b<a$.

We will omit most of the details since this case is very similar to the previous ones. According to Notation II.1, we set $\mathfrak{h}=\left\{\operatorname{diag}\left(\left[\begin{array}{cc}0 & \theta_{1} \\ -\theta_{1} & 0\end{array}\right], \ldots,\left[\begin{array}{cc}0 & \theta_{n} \\ -\theta_{n} & 0\end{array}\right], 0\right): \theta_{i} \in \mathbb{C} \forall i\right\}$, $\varepsilon_{i}\left(\operatorname{diag}\left(\left[\begin{array}{cc}0 & \theta_{1} \\ -\theta_{1} & 0\end{array}\right], \ldots,\left[\begin{array}{cc}0 & \theta_{n} \\ -\theta_{n} & 0\end{array}\right], 0\right)\right)=\theta_{i}$ for each $1 \leq i \leq n, \Sigma^{+}(\mathfrak{g}, \mathfrak{h})=\left\{\varepsilon_{i} \pm \varepsilon_{j}: i<j\right\} \cup\left\{\varepsilon_{i}\right\}$,

$$
\begin{aligned}
P(\mathfrak{g}) & =\left\{\sum_{i} a_{i} \varepsilon_{i}: a_{i} \in \mathbb{Z} \forall i, \text { or } a_{i}-1 / 2 \in \mathbb{Z} \forall i\right\}, & P(G) & =\mathbb{Z} \varepsilon_{1} \oplus \cdots \oplus \mathbb{Z} \varepsilon_{n}, \\
P^{++}(\mathfrak{g}) & =\left\{\sum_{i} a_{i} \varepsilon_{i} \in P(\mathfrak{g}): a_{1} \geq a_{2} \geq \cdots \geq a_{n} \geq 0\right\}, & P^{++}(G) & =P^{++}(\mathfrak{g}) \cap P(G) .
\end{aligned}
$$


It is well known that (see Exercises IV.10 and V.8 in Ref. 16)

$$
\operatorname{Sym}^{k}\left(\mathbb{C}^{2 n+1}\right) \simeq \pi_{k \omega_{1}} \oplus \operatorname{Sym}^{k-2}\left(\mathbb{C}^{2 n+1}\right), \quad \pi_{\widetilde{\omega}_{p}} \simeq \bigwedge^{p}\left(\mathbb{C}^{2 n+1}\right),
$$

where $\mathbb{C}^{2 n+1}$ denotes the standard representation of $\mathfrak{g}$. Actually, $\pi_{k \omega_{1}}$ can be realized inside $\operatorname{Sym}^{k}\left(\mathbb{C}^{2 n+1}\right)$ as the subspace of harmonic homogeneous polynomials of degree $k$.

Lemma V.3. Let $n \geq 2, \mathfrak{g}=\mathfrak{s o}(2 n+1, \mathbb{C}), G=\mathrm{SO}(2 n+1), k \geq 0$, and $1 \leq p \leq n$. For $\mu=\sum_{j=1}^{n} a_{j} \varepsilon_{j}$ $\in P(G)$, we have that

$$
\begin{aligned}
& m_{\pi_{k \omega_{1}}}(\mu)=m_{\pi_{k \varepsilon_{1}}}(\mu)=\left(\begin{array}{c}
r(\mu)+n-1 \\
n-1
\end{array}\right) \quad \text { where } r(\mu)=\left\lfloor\frac{k-\|\mu\|_{1}}{2}\right\rfloor, \\
& m_{\pi_{\widetilde{\omega}_{p}}}(\mu)=\left\{\begin{array}{ll}
\left(\begin{array}{c}
n-p+r(\mu) \\
\lfloor r(\mu) / 2\rfloor
\end{array}\right) & \text { if }\left|a_{j}\right| \leq 1 \forall j, \\
0 & \text { otherwise, }
\end{array} \text { where } r(\mu)=p-\|\mu\|_{1} .\right.
\end{aligned}
$$

Proof. Let $\mathcal{P}_{k}$ be the space of complex homogeneous polynomials of degree $k$ in the variables $x_{1}, \ldots, x_{2 n+1}$. Set $f_{j}=x_{2 j-1}+i x_{2 j}$ and $g_{j}=x_{2 j-1}-i x_{2 j}$ for $1 \leq j \leq n$. One can check that the polynomials $f_{1}^{k_{1}} \ldots f_{n}^{k_{n}} g_{1}^{l_{1}} \ldots g_{n}^{l_{n}} x_{2 n+1}^{k_{0}}$ with $k_{0}, \ldots, k_{n}, l_{1}, \ldots, l_{n}$ non-negative integers satisfying that $\sum_{j=0}^{n} k_{j}+\sum_{j=1}^{n} l_{j}=k$ form a basis of $\mathcal{P}_{k}$ given by weight vectors, each of them of weight $\mu=\sum_{j=1}^{n}\left(k_{j}-l_{j}\right) \varepsilon_{j}$. Notice that the number $k_{0}$ does not take part of $\mu$.

Consequently, $m_{\pi_{\mathcal{P}_{k}}}(\mu)$ for $\mu=\sum_{j=1}^{n} a_{j} \varepsilon_{j}$ is the number of tuples $\left(k_{0}, \ldots, k_{n}, l_{1}, \ldots, l_{n}\right) \in \mathbb{N}_{0}^{2 n+1}$ satisfying $a_{j}=k_{j}-l_{j}$ for all $1 \leq j \leq n$ and

$$
\sum_{j=0}^{n} k_{j}+\sum_{j=1}^{n} l_{j}=k .
$$

Note that (21) implies $k-\|\mu\|_{1}-k_{0}=2 s$ for some integer $s \geq 0$.

We fix an integer $s$ satisfying $0 \leq s \leq r:=\left\lfloor\left(k-\|\mu\|_{1}\right) / 2\right\rfloor$. Set $k_{0}=k-\|\mu\|_{1}-2 s \geq 0$. As in the proof of Lemma III.2, the number of $\left(k_{1}, \ldots, k_{n}, l_{1}, \ldots, l_{n}\right) \in \mathbb{N}_{0}^{2 n}$ satisfying that $a_{j}=k_{j}-l_{j}$ for all 1 $\leq j \leq n$ and (21) is equal to $\left(\begin{array}{c}s+n-1 \\ n-1\end{array}\right)$. Hence,

$$
m_{\mathcal{P}_{k}}(\mu)=\sum_{s=0}^{r}\left(\begin{array}{c}
s+n-1 \\
n-1
\end{array}\right)=\left(\begin{array}{c}
r+n \\
n
\end{array}\right)
$$

The second equality is well known. It may be proven by showing that both sides are the $r$-term of the generating function $(1-z)^{-(n+1)}$. From (18), we conclude that $m_{\pi_{k \varepsilon_{1}}}(\mu)=m_{\mathcal{P}_{k}}(\mu)-m_{\mathcal{P}_{k-2}}(\mu)$ $=\left(\begin{array}{c}r+n \\ n\end{array}\right)-\left(\begin{array}{c}r-1+n \\ n\end{array}\right)=\left(\begin{array}{c}r+n-1 \\ n-1\end{array}\right)$.

We have that $\pi_{\widetilde{\omega}_{p}} \simeq \bigwedge^{p}\left(\mathbb{C}^{2 n+1}\right)$ by (18). By setting $v_{j}=e_{2 j-1}-i e_{2 j}, v_{j+n}=e_{2 j-1}+i e_{2 j}$ and $v_{2 n+1}$ $=e_{2 n+1}$, one obtains that the vectors $w_{I}:=v_{i_{1}} \wedge \cdots \wedge v_{i_{p}}$, for $I=\left\{i_{1}, \ldots, i_{p}\right\}$ satisfying $1 \leq i_{1}<\cdots$ $<i_{p} \leq 2 n+1$, form a basis of $\bigwedge^{p}\left(\mathbb{C}^{2 n+1}\right)$. Furthermore, $w_{I}$ is a weight vector of weight $\mu=\sum_{j=1}^{n} a_{j} \varepsilon_{j}$ given by (11). Note that the condition of $2 n+1$ being or not in $I$ does not influence on $\mu$.

Hence, $\mu=\sum_{j} a_{j} \varepsilon_{j}$ is a weight of $\bigwedge^{p}\left(\mathbb{C}^{2 n+1}\right)$ if and only if $\left|a_{j}\right| \leq 1$ for all $j$ and $p-\|\mu\|_{1} \geq$ 0 . Proceeding as in Lemma III.2, by writing $s=\left\lfloor\frac{p-\|\mu\|_{1}}{2}\right\rfloor \geq 0$, the multiplicity of $\mu$ is $\left(\begin{array}{c}n-p+2 s \\ s\end{array}\right)$ if $p-\|\mu\|_{1}$ is even and $\left(\begin{array}{c}n-p+2 s+1 \\ s\end{array}\right)$ if $p-\|\mu\|_{1}$ is odd.

Theorem V.4. Let $\mathfrak{g}=\mathfrak{s v}(2 n+1, \mathbb{C})$ and $G=\mathrm{SO}(2 n+1)$ for some $n \geq 2$ and let $k \geq 0$ an integer. Let $\mu \in P(\mathfrak{g}) \backslash P(G)$. Write $r(\mu)=k+\frac{n}{2}-\|\mu\|_{1}$, then

$$
m_{\pi_{k \omega_{1}+\omega_{n}}}(\mu)=\left(\begin{array}{c}
r(\mu)+n-1 \\
n-1
\end{array}\right) \text {. }
$$

Furthermore, $m_{k \omega_{1}+\omega_{n}}(\mu)=0$ for all $\mu \in P(G)$.

Proof. This proof is very similar to Lemma 4.2 in Ref. 4. The assertion $m_{k \omega_{1}+\omega_{n}}(\mu)=0$ for every $\mu \in P(G)$ is clear since any weight of $\pi_{k \omega_{1}+\omega_{n}}$ is equal to the highest weight $k \omega_{1}+\omega_{n}$ minus a sum of positive roots, which clearly lies in $P(\mathfrak{g}) \backslash P(G)$. 
Let $\mu \in P(\mathfrak{g}) \backslash P(G)$. We may assume that $\mu$ is dominant, thus $\mu=\frac{1}{2} \sum_{i=1}^{n} a_{i} \varepsilon_{i}$ with $a_{1} \geq \cdots \geq a_{n}$ $\geq 1$ odd integers. One has that

$$
\pi_{k \omega_{1}} \otimes \pi_{\omega_{n}} \simeq \pi_{k \omega_{1}+\omega_{n}} \oplus \pi_{(k-1) \omega_{1}+\omega_{n}},
$$

for any $k \geq 1$. Indeed, it follows immediately by Exercise V.19 in Ref. 16 since in its sum over the weights of $\pi_{\omega_{n}}$, the only non-zero terms are attained at the weights $\omega_{n}$ and $\omega_{n}-\omega_{1}$.

It is well known that the set of weights of $\pi_{\omega_{n}}$ is $\mathcal{P}\left(\pi_{\omega_{n}}\right):=\left\{\frac{1}{2} \sum_{i=1}^{n} b_{i} \varepsilon_{i}:\left|b_{i}\right|=1\right\}$ and $m_{\pi_{\omega_{n}}}(v)=1$ for all $v \in \mathcal{P}\left(\pi_{\omega_{n}}\right)$ (see, for instance, Exercise V.35 in Ref. 16).

We proceed now to prove (22) by induction on $k$. It is clear for $k=0$ by the previous paragraph. Suppose that it holds for $k-1$. By this assumption and (23), we obtain that

$$
m_{\pi_{k \omega_{1}+\omega_{n}}}(\mu)=m_{\pi_{k \omega_{1}} \otimes \pi_{\omega_{n}}}(\mu)-m_{\pi_{(k-1) \omega_{1}+\omega_{n}}}(\mu)=m_{\pi_{k \omega_{1}} \otimes \pi_{\omega_{n}}}(\mu)-\left(\begin{array}{c}
r+n-2 \\
n-1
\end{array}\right),
$$

where $r=k+\frac{n}{2}-\|\mu\|_{1}$. It only remains to prove that $m_{\pi_{k \omega_{1}} \otimes \pi_{\omega_{n}}}(\mu)=\left(\begin{array}{c}r+n-1 \\ n-1\end{array}\right)+\left(\begin{array}{c}r+n-2 \\ n-1\end{array}\right)$.

Similarly to (3), we have that $m_{\pi_{k \omega_{1}} \otimes \pi_{\omega_{n}}}(\mu)=\sum_{\eta \in \mathcal{P}\left(\pi_{\omega_{n}}\right)} m_{\pi_{k \omega_{1}}}(\mu-\eta)$. Since $\mu$ is dominant, for any $\eta=\frac{1}{2} \sum_{i=1}^{n} b_{i} \varepsilon_{i} \in \mathcal{P}\left(\pi_{\omega_{n}}\right)$, it follows that

$$
\|\mu-\eta\|_{1}=\frac{1}{2} \sum_{i=1}^{n}\left(a_{i}-b_{i}\right)=\|\mu\|_{1}+\frac{n}{2}-\ell_{1}(\eta)=k-r+n-\ell_{1}(\eta),
$$

where $\ell_{1}(\eta)=\#\left\{1 \leq i \leq n: b_{i}=1\right\}$. By Lemma V.3, $m_{\pi_{k \omega_{1}}}(\mu-\eta) \neq 0$ only if $r+\ell_{1}(\eta)-n \geq 0$. For each integer $\ell_{1}$ satisfying $n-r \leq \ell_{1} \leq n$, there are $\left(\begin{array}{l}n \\ \ell_{1}\end{array}\right)$ weights $\eta \in \mathcal{P}\left(\pi_{\omega_{n}}\right)$ such that $\ell_{1}(\eta)=\ell_{1}$. On account of the above remarks,

$$
m_{\pi_{k \omega_{1}} \otimes \pi_{\omega_{n}}}(\mu)=\sum_{\ell_{1}=n-r}^{n}\left(\begin{array}{c}
\left\lfloor\frac{r+\ell_{1}-n}{2}\right\rfloor+n-1 \\
n-1
\end{array}\right)\left(\begin{array}{l}
n \\
\ell_{1}
\end{array}\right)=\sum_{j=0}^{r}\left(\begin{array}{c}
\left\lfloor\frac{r-j}{2}\right\rfloor+n-1 \\
n-1
\end{array}\right)\left(\begin{array}{l}
n \\
j
\end{array}\right) .
$$

We claim that the last term in (25) equals $\left(\begin{array}{c}r+n-1 \\ n-1\end{array}\right)+\left(\begin{array}{c}r+n-2 \\ n-1\end{array}\right)$. Indeed, a simple verification shows that both numbers are the $r$ th term of the generating function $\frac{1+z}{(1-z)^{n}}$. From (24) and (25), we conclude that $m_{\pi_{k \omega_{1}+\omega_{n}}}(\mu)=\left(\begin{array}{c}r+n-1 \\ n-1\end{array}\right)$ as asserted.

Lemma V.5. Let $n \geq 2, \mathfrak{g}=\mathfrak{s o}(2 n+1, \mathbb{C}), G=\mathrm{SO}(2 n+1), k \geq 0,1 \leq p<n$, and $\mu \in P(G)$. Write $r(\mu)=k+p-\|\mu\|_{1}$. Then

$$
\begin{aligned}
m_{\sigma_{k, p}}(\mu)= & \sum_{t=0}^{\lfloor p / 2\rfloor}\left(\begin{array}{c}
n-p+2 t \\
t
\end{array}\right) \sum_{\beta=0}^{p-2 t} 2^{p-2 t-\beta}\left(\begin{array}{c}
n-Z(\mu) \\
\beta
\end{array}\right)\left(\begin{array}{c}
Z(\mu) \\
p-2 t-\beta
\end{array}\right) \sum_{\alpha=0}^{\beta}\left(\begin{array}{c}
\beta \\
\alpha
\end{array}\right)\left(\begin{array}{c}
\left\lfloor\frac{r(\mu)}{2}\right\rfloor-p+\alpha+t+n-1 \\
n-1
\end{array}\right) \\
& +\sum_{t=0}^{\lfloor(p-1) / 2\rfloor}\left(\begin{array}{c}
n-p+1+2 t \\
t
\end{array}\right) \sum_{\beta=0}^{p-1-2 t} 2^{p-1-2 t-\beta}\left(\begin{array}{c}
n-Z(\mu) \\
\beta
\end{array}\right)\left(\begin{array}{c}
Z(\mu) \\
p-1-2 t-\beta
\end{array}\right) \\
& \sum_{\alpha=0}^{\beta}\left(\begin{array}{l}
\beta \\
\alpha
\end{array}\right)\left(\begin{array}{c}
\left\lfloor\frac{r(\mu)+1}{2}\right\rfloor-p+\alpha+t+n-1 \\
n-1
\end{array}\right) .
\end{aligned}
$$

Proof. Write $r=k+p-\|\mu\|_{1}$ and $\ell=Z(\mu)$ and assume $\mu$ dominant. Define $\mathcal{P}_{t, \beta, \alpha}^{(p)}$ as in (12). From Lemma V.3, we deduce that the set of weights of $\pi_{\widetilde{\omega}_{p}}$ is

$$
\mathcal{P}\left(\pi_{\widetilde{\omega}_{p}}\right):=\left(\bigcup_{t=0}^{\lfloor p / 2\rfloor} \bigcup_{\beta=0}^{p-2 t} \bigcup_{\alpha=0}^{\beta} \mathcal{P}_{t, \beta, \alpha}^{(p)}\right) \cup\left(\bigcup_{t=0}^{\lfloor p-1 / 2\rfloor} \bigcup_{\beta=0}^{p-1-2 t} \bigcup_{\alpha=0}^{\beta} \mathcal{P}_{t, \beta, \alpha}^{(p-1)}\right) .
$$


This fact and (3) give

$$
\begin{aligned}
m_{\sigma_{k, p}}(\mu)= & \sum_{t=0}^{\lfloor p / 2\rfloor} \sum_{\beta=0}^{p-2 t} \sum_{\alpha=0}^{\beta}\left(\begin{array}{c}
\left\lfloor\frac{r}{2}\right\rfloor+t+\alpha-p+n-1 \\
n-1
\end{array}\right)\left(\begin{array}{c}
n-p+2 t \\
t
\end{array}\right) \# \mathcal{P}_{t, \beta, \alpha}^{(p)} \\
& +\sum_{t=0}^{\lfloor(p-1) / 2\rfloor} \sum_{\beta=0}^{p-1-2 t} \sum_{\alpha=0}^{\beta}\left(\begin{array}{c}
\left\lfloor\frac{r-1}{2}\right\rfloor+t+\alpha-p+n \\
n-1
\end{array}\right)\left(\begin{array}{c}
n-p+1+2 t \\
t
\end{array}\right) \# \mathcal{P}_{t, \beta, \alpha}^{(p-1)},
\end{aligned}
$$

since $\|\mu-\eta\|_{1}=k-r-2(t+\alpha-p)$ for all $\eta \in \mathcal{P}_{t, \beta, \alpha}^{(p)}$ and $\|\mu-\eta\|_{1}=k-r-2(t+\alpha-p)-1$ for all $\eta \in \mathcal{P}_{t, \beta, \alpha}^{(p-1)}$. The proof follows by (13).

Lemmas II.3 and V.5 complete the proof of Theorem V.1.

\section{TYPE A}

Type $\mathrm{A}_{n}$ is the simplest case to compute the weight multiplicity formula of $\pi_{k, p}$. Actually, it follows immediately by standard calculations using Young diagrams. We include this formula to complete the list of all classical simple Lie algebras.

We consider in $\mathfrak{g}=\mathfrak{s l}(n+1, \mathbb{C}), \mathfrak{h}=\left\{\operatorname{diag}\left(\theta_{1}, \ldots, \theta_{n+1}\right): \theta_{i} \in \mathbb{C} \forall i, \sum_{i=1}^{n+1} \theta_{i}=0\right\}$. We set $\varepsilon_{i}\left(\operatorname{diag}\left(\theta_{1}, \ldots, \theta_{n+1}\right)\right)=\theta_{i}$ for each $1 \leq i \leq n+1$. We will use the conventions of Ref. 13 in Lecture 15. Thus

$$
\mathfrak{h}^{*}=\bigoplus_{i=1}^{n+1} \mathbb{C} \varepsilon_{i} /\left\langle\sum_{i=1}^{n+1} \varepsilon_{i}=0\right\rangle,
$$

the set of positive roots is $\Sigma^{+}(\mathfrak{g}, \mathfrak{h})=\left\{\varepsilon_{i}-\varepsilon_{j}: 1 \leq i<j \leq n+1\right\}$, and the weight lattice is $P(\mathfrak{g})$ $=\bigoplus_{i=1}^{n+1} \mathbb{Z} \varepsilon_{i} /\left\langle\sum_{i=1}^{n+1} \varepsilon_{i}=0\right\rangle$. By abuse of notation, we use the same letter $\varepsilon_{i}$ for the image of $\varepsilon_{i}$ in $\mathfrak{h}^{*}$. A weight $\mu=\sum_{i=1}^{n+1} a_{i} \varepsilon_{i}$ is dominant if $a_{1} \geq a_{2} \geq \cdots \geq a_{n+1}$.

The representations having highest weights $\lambda=\sum_{i=1}^{n+1} a_{i} \varepsilon_{i}$ and $\mu=\sum_{i=1}^{n+1} b_{i} \varepsilon_{i}$ are isomorphic if and only if $a_{i}-b_{i}$ is constant, independent of $i$. Consequently, we can restrict to those $\lambda=\sum_{i=1}^{n+1} a_{i} \varepsilon_{i}$ with $a_{n+1}=0$. Then,

$$
P^{++}(\mathfrak{g})=\left\{\sum_{i=1}^{n} a_{i} \varepsilon_{i} \in P(\mathfrak{g}): a_{1} \geq a_{2} \geq \cdots \geq a_{n} \geq 0\right\} .
$$

The corresponding fundamental weights are given by $\omega_{p}=\varepsilon_{1}+\cdots+\varepsilon_{p}$ for each $1 \leq p \leq n$.

It is well known that for $\lambda \in P^{++}(\mathrm{g})$ and $\mu$ a weight of $\pi_{\lambda}$, one can assume that $\mu=\sum_{i=1}^{n+1} a_{i} \varepsilon_{i}$ with $a_{i} \in \mathbb{N}_{0}$ for all $i$ and $\sum_{i=1}^{n+1} a_{i}=\|\lambda\|_{1}$.

Theorem VI.1. Let $\mathfrak{g}=\mathfrak{s l}(n+1, \mathbb{C})$ for some $n \geq 1$ and let $k \geq 0,1 \leq p \leq n$ integers. Let $\mu=\sum_{i=1}^{n+1} a_{i} \varepsilon_{i} \in P(\mathfrak{g})$ with $a_{i} \in \mathbb{N}_{0}$ for all $i$ and $\sum_{i=1}^{n+1} a_{i}=k+p$. If $a_{1}+a_{2}+\cdots+a_{j} \leq k+j$ for all 1 $\leq j \leq p$, then

$$
m_{\pi_{k \omega_{1}+\omega_{p}}}(\mu)=\left(\begin{array}{c}
n-Z(\mu) \\
p-1
\end{array}\right)
$$

and $m_{\pi_{k, p}}(\mu)=0$ otherwise.

Proof. The Young diagram corresponding to the representation $\pi_{k \omega_{1}+\omega_{p}}$ is the diagram with $p$ rows, having all length 1 , excepting the first one which has length $k+1$. It is well known that the multiplicity of the weight $\mu$ in this representation is equal to the number of ways one can fill its Young diagram with $a_{1} 1$ 's, $a_{2} 2$ 's, $\ldots, a_{n+1}(n+1)$ 's, in such a way that the entries in the first row are non-decreasing and those in the first column are strictly increasing (see, for instance, Sec. 15.3 in Ref. 13).

Consequently, the multiplicity of $\mu$ is equal to the number of ways of filling the first column. Since the first entry is uniquely determined, one has to choose $p-1$ different numbers for the rest of the entries. Hence, the theorem follows. 


\section{CONCLUDING REMARKS}

For a classical complex Lie algebra $\mathfrak{g}$, it has been shown a closed explicit formula for the weight multiplicities of a representation in any $p$-fundamental string, namely, any irreducible representation of $\mathfrak{g}$ having highest weight $k \omega_{1}+\omega_{p}$, for some integers $k \geq 0$ and $1 \leq p \leq n$. When $\mathfrak{g}$ is of type $\mathrm{A}_{n}$, the proof was quite simple and the corresponding formula could be probably established from a more general result. To the best of the authors' knowledge, the obtained expressions of the weight multiplicities for types $\mathrm{B}_{n}, \mathrm{C}_{n}$, and $\mathrm{D}_{n}$ are new, except for small values of $n$, probably $n \leq 3$.

Although the formulas in Theorems III.1, IV.1, and V.1 (types $\mathrm{C}_{n}, \mathrm{D}_{n}$, and $\mathrm{B}_{n}$, respectively) look complicated and long, they are easily handled in practice. It is important to note that all sums are over (integer) intervals, without including any sum over partitions or permutations. Furthermore, there are only combinatorial numbers in each term. Consequently, it is a simple matter to implement them in a computer program, obtaining a very fast algorithm even when the rank $n$ of the Lie algebra is very large.

Moreover, for $p$ and a weight $\mu$ fixed, the formulas become a quasi-polynomial on $k$. This fact was already predicted and follows by the Kostant multiplicity formula, such as M. Vergne pointed out to Kumar and Prasad ${ }^{20}$ (see also Refs. 3 and 27).

For instance, when $\mathfrak{g}=\mathfrak{s} \mathfrak{p}(2 n, \mathbb{C})\left(\right.$ type $\left.\mathrm{D}_{n}\right)$, Theorem IV.1 ensures that

$$
m_{\pi_{k \omega_{1}}}(\mu)= \begin{cases}\left(\frac{k-\|\mu\|_{1}}{2^{n}+n-2}\right) & \text { if } k \geq\|\mu\|_{1} \text { and } k \equiv\|\mu\|_{1} \quad(\bmod 2), \\ 0 & \text { otherwise. }\end{cases}
$$

Consequently, the generating function encoding the numbers $\left\{m_{\pi_{k \omega_{1}}}(\mu): k \geq 0\right\}$ is a rational function. Indeed,

$$
\sum_{k \geq 0} m_{\pi_{k \omega_{1}}}(\mu) z^{k}=\sum_{k \geq 0} m_{\pi_{\left(2 k+\|\mu\|_{1}\right) \omega_{1}}}(\mu) z^{2 k+\|\mu\|_{1}}=\frac{z^{\|\mu\|_{1}}}{\left(1-z^{2}\right)^{n-1}} .
$$

From a different point of view, for fixed integers $k$ and $p$, the formulas are quasi-polynomials in the variables $\|\mu\|_{1}$ and $Z(\mu)$.

We end the article with a summary of past (and possible future) applications of multiplicity formulas in spectral geometry. We consider a locally homogeneous space $\Gamma \backslash G / K$ with the (induced) standard metric, where $G$ is a compact semisimple Lie group, $K$ is a closed subgroup of $G$, and $\Gamma$ is a finite subgroup of the maximal torus $T$ of $G$. When $G=\operatorname{SO}(2 n), K=\operatorname{SO}(2 n-1)$, and $\Gamma$ is cyclic acting freely on $G / K \simeq S^{2 n-1}$, we obtain a lens space.

In order to determine explicitly the spectrum of a (natural) differential operator acting on smooth sections of a (natural) vector bundle on $\Gamma \backslash G / K$ (e.g., Laplace-Beltrami operator, Hodge-Laplace operator on $p$-form, Dirac operator), one has to calculate-among other things-numbers of the form $\operatorname{dim} V_{\pi}^{\Gamma}$ for $\pi$ in a subset of the unitary dual $\widehat{G}$ depending on the differential operator. Since $\Gamma \subset T, \operatorname{dim} V_{\pi}^{\Gamma}$ can be computed by counting the $\Gamma$-invariant weights in $\pi$ according to its multiplicity, so the problem is reduced to know $m_{\pi}(\mu)$.

At the moment, some weight multiplicity formulas have been successfully applied to the problem described above. The multiplicity formula for $\pi_{k \omega_{1}}$ in type $\mathrm{D}_{n}$ (Lemma IV.3) was used by Miatello, Rossetti, and the first named author ${ }^{23}$ to determine the spectrum of the Laplace-Beltrami operator on a lens space. Furthermore, Corollary I.1 for type $\mathrm{D}_{n}$ was shown in the same article (Lemma 3.3) obtaining a characterization of lens spaces $p$-isospectral for all $p$ (i.e., their Hodge-Laplace operators on $p$-forms have the same spectra). Later, Boldt and the first named author ${ }^{4}$ considered in the Dirac operator on odd-dimensional spin lens spaces. In this work, it was obtained and used in Theorem IV.2, namely, the multiplicity formula for type $\mathrm{D}_{n}$ of the spin representations $\pi_{k \omega_{1}+\omega_{n-1}}$ and $\pi_{k \omega_{1}+\omega_{n}}$.

As a continuation of the study begun in Ref. 23, Theorem IV.1 was applied in the preprint ${ }^{22}$ to determine explicitly every $p$-spectra of a lens space. Here, as usual, $p$-spectrum stands for the spectrum of the Hodge-Laplace operator acting on smooth $p$-forms. This article was the motivation to write the present paper.

The remaining formulas in the article may be used with the same goal. Actually, any application of the formulas for type $\mathrm{D}_{n}$ can be translated to an analogue application for type $\mathrm{B}_{n-1}$, working in spaces covered by $S^{2 n-2}$ in place of $S^{2 n-1}$ (Sec. 4 in Ref. 15). This was partially done ${ }^{21}$ by applying 
Lemma V.3. The result extends Ref. 23 (for the Laplace-Beltrami operator) to even-dimensional lens orbifolds.

A different but feasible application can be done for type $\mathrm{A}_{n}$. One may consider the complex projective space $P^{n}(\mathbb{C})=\mathrm{SU}(n+1) / S(\mathrm{U}(n) \times \mathrm{U}(1))$. However, more general representations must be used. Indeed, in Ref. 21, it was considered the Laplace-Beltrami operator and the representations involved had highest weights $k\left(\omega_{1}+\omega_{n}\right)$ for $k \geq 0$.

Theorem III.1 (type $\mathrm{C}_{n}$ ) does not have an immediate application since the spherical representations of the symmetric space $\operatorname{Sp}(n) /(\operatorname{Sp}(n-1) \times \operatorname{Sp}(1))$ have highest weight of the form $k \omega_{2}$ for $k \geq 0$. Maddox ${ }^{26}$ obtained a multiplicity formula for these representations. However, this expression is not explicit enough to be applied in this problem. An exception was the case $n=2$, since in Ref. 21, it was applied the closed multiplicity formula in Ref. 5. It is not know by the authors if there is a closed subgroup $K$ of $G=\operatorname{Sp}(n)$ such that the spherical representations of $G / K$ are $\pi_{k \omega_{1}}$ for $k$ $\geq 0$, that is,

$$
\left\{\pi \in \widehat{G}: V_{\pi}^{K} \simeq \operatorname{Hom}_{K}\left(V_{\pi}, \mathbb{C}\right) \neq 0\right\}=\left\{\pi_{k \omega_{1}}: k \geq 0\right\} .
$$

In such a case, Theorem III.1 could be used.

\section{ACKNOWLEDGMENTS}

The authors wish to thank the anonymous referee for carefully reading the article and giving them helpful comments. This research was partially supported by grants from CONICET, FONCyT, and SeCyT-UNC. The first named author was supported by the Alexander von Humboldt Foundation.

${ }^{1}$ Baldoni, M. W., Beck, M., Cochet, C., and Vergne, M., "Volume computation for polytopes and partition functions for classical root systems," Discrete Comput. Geom. 35(4), 551-595 (2006).

${ }^{2}$ Bliem, T., "On weight multiplicities of complex simple Lie algebras," Ph.D. thesis, Universität zu Köln, MathematischNaturwissenschaftliche Fakultät, 2008.

${ }^{3}$ Bliem, T., "Chopped and sliced cones and representations of Kac-Moody algebras," J. Pure Appl. Algebra 214(7), 1152-1164 (2016).

${ }^{4}$ Boldt, S. and Lauret, E. A., “An explicit formula for the Dirac multiplicities on lens spaces,” J. Geom. Anal. 27, 689-725 (2017).

${ }^{5}$ Cagliero, L. and Tirao, P., "A closed formula for weight multiplicities of representations of $\mathrm{Sp}_{2}(\mathbb{C})$," Manuscripta Math. 115(4), 417-426 (2004).

${ }^{6}$ Cavallin, M., "An algorithm for computing weight multiplicities in irreducible modules for complex semisimple Lie algebras," J. Algebra 471, 492-510 (2017).

${ }^{7}$ Cochet, C., "Vector partition function and representation theory," in Conference Proceedings on Formal Power Series and Algebraic Combinatorics, Taormina, Italy (Sém. Lothar. Combin., 2007), Vol. B54Al, pp. 1009-1020.

${ }^{8}$ Fernández Núñez, J., García Fuertes,W., and Perelomov, A. M., "On an approach for computing the generating functions of the characters of simple Lie algebras," J. Phys. A: Math. Theor. 47(14), 091702 (2014).

${ }^{9}$ Fernández Núñez, J., García Fuertes,W., and Perelomov, A. M., "On the generating function of weight multiplicities for the representations of the Lie algebra $\mathrm{C}_{2}$," J. Math. Phys. 56(4), 041702 (2015).

${ }^{10}$ Fernández Núñez, J., García Fuertes,W., and Perelomov, A. M., "Generating functions and multiplicity formulas: The case of rank two simple Lie algebras," J. Math. Phys. 56(9), 091702 (2015).

${ }^{11}$ Fernández Núñez, J., García Fuertes,W., and Perelomov, A. M., "Some results on generating functions for characters and weight multiplicities of the Lie algebra $\mathrm{A}_{3}$," e-print arXiv:1705.03711v1 (2017).

${ }^{12}$ Freudenthal, H., "Zur berechnung der charaktere der halbeinfachen Lieschen gruppen. I,” Indag. Math. 57(369-376), 487491 (1954).

${ }^{13}$ Fulton, W. and Harris, J., Representation Theory: A First Course (Springer-Verlag, New York, 2004).

${ }^{14}$ Harris, P., "Combinatorial problems related to Kostant's weight multiplicity formula," Ph.D. thesis, University of WisconsinMilwaukee, Milwaukee, WI, 2012.

${ }^{15}$ Ikeda, A. and Taniguchi, Y., "Spectra and eigenforms of the Laplacian on $S^{n}$ and $P^{n}(\mathbb{C})$, ," Osaka J. Math. 15(3), 515-546 (1978).

${ }^{16}$ Knapp, A. W., Lie Groups Beyond an Introduction, Progress in Mathematics (Birkhäuser Boston, Inc., 2002), Vol. 140.

${ }^{17}$ Koike, K., "On new multiplicity formulas of weights of representations for the classical groups," J. Algebra 107, 512-533 (1987).

${ }^{18}$ Koike, K. and Terada, I., "Young-diagrammatic methods for the representation theory of the classical groups of type $B_{n}$, $C_{n}, D_{n}$, , J. Algebra 107, 466-511 (1987).

${ }^{19}$ Kostant, B., "A formula for the multiplicity of a weight," Trans. Am. Math. Soc. 93(1), 53-73 (1959).

${ }^{20}$ Kumar, S. and Prasad, D., "Dimension of zero weight space: An algebro-geometric approach,” J. Algebra 403, 324-344 (2014).

${ }^{21}$ Lauret, E. A., "Spectra of orbifolds with cyclic fundamental groups," Ann. Global Anal. Geom. 50(1), 1-28 (2016).

${ }^{22}$ Lauret, E. A., "The spectrum on p-forms of a lens space," preprint arXiv:1604.02471v4 (2016). 
${ }^{23}$ Lauret, E. A., Miatello, R. J., and Rossetti, J. P., “Spectra of lens spaces from 1-norm spectra of congruence lattices,” Int. Math. Res. Not. 2016(4), 1054-1089.

${ }^{24}$ Littelmann, P., "Paths and root operators in representation theory," Ann. Math. 142(3), 499-525 (1995).

${ }^{25}$ Lusztig, G., "Singularities, character formulas, and a q-analog of weight multiplicities," Astérisque 101-102, 208-229 (1983).

${ }^{26}$ Maddox, J., "An elementary approach to weight multiplicities in bivariate irreducible representations of $\operatorname{Sp}(2 r)$," Commun. Algebra 42(9), 4094-4101 (2014).

${ }^{27}$ Meinrenken, E. and Sjamaar, R., "Singular reduction and quantization,” Topology 38(4), 699-762 (1999).

${ }^{28}$ Sahi, S., "A new formula for weight multiplicities and characters," Duke Math. J 101(1), 77-84 (2000).

${ }^{29}$ Schützer,W., "On some combinatorial aspects of representation theory," Ph.D. thesis, Rutgers The State University of New Jersey, 2004.

${ }^{30}$ Schützer,W., "A new character formula for Lie algebras and Lie groups," J. Lie Theory 22(3), 817-838 (2012).

${ }^{31}$ Stein, W. A. et al., Sage Mathematics Software (Version 4.3). The Sage Development Team, 2009, www.sagemath.org. 